\title{
Effects of Medial Preoptic Lesions on Placentophagia and on the Onset of Maternal Behavior in the Rat $^{1}$
}

\author{
MICHAEL NOONAN AND MARK B. KRISTAL ${ }^{2}$ \\ Department of Psychology, State University of New York at Buffalo, Buffalo, NY 14226
}

(Received 10 January 1979)

\begin{abstract}
NOONAN, M. AND M. B. KRISTAL. Effects of medial preoptic lesions on placentophagia and on the onset of maternal behavior in the rat. PHYSIOL. BEHAV. 22(6) 1197-1202, 1979.- Lesions of the medial preoptic area (MPO) were produced through permanently indwelling electrodes $24 \mathrm{hr}$ prior to parturition in pregnant rats, or $24 \mathrm{hr}$ prior to donor-placenta presentation in virgin rats determined in a pretest to be placentophages. The lesions had no disruptive effect on placentophagia in the virgin females. However, MPO lesions did delay the onset of placentophagia, pup-retrieval, and nestbuilding in some parturient rats. In others, lesions produced an impairment (in latency and quality) only of nest-building. None showed any impairment of pup-licking, or in the clear tendency to leave excreted waste away from the gathered pups. These results suggest the possibility of at least semi-independent mechanisms for the various components of maternal behavior.
\end{abstract}

Medial preoptic area Hypothalamus Maternal behavior Placentophagia Parturition Lesions

NUMAN [7] reported that lesions in the medial preoptic area, or knife cuts along the lateral border of that area, disrupt already established maternal behavior in the rat. He demonstrated that these procedures, performed on Day 5 postpartum, either suppressed or eliminated subsequent pup retrieval, nest-building, and nursing. Numan, Rosenblatt, and Komisaruk [8] reported that in nulliparous virgin rats, medial preoptic lesions also suppressed the initiation of maternal behavior produced by continuous exposure to rat pups.

Neither the maintenance of maternal behavior [11] nor the pup-induced initiation of maternal behavior in virgins [15] depends on ovarian hormones. On the other hand the very rapid onset of maternal responsiveness to pups shown by parturient rats is in large part hormonally triggered $[5,15]$ and estrogen has been implicated as playing an important role $[12,13]$. The medial preoptic area has been shown to contain estrogen-concentrating neurons [10], and Numan et al. [8], provided evidence that that area also plays a role in hormonally-induced maternal behavior, in that estrogen implants there, in hysterectomized-ovariectomized Day-16 pregnant rats, resulted in shorter latencies to maternal behavior than did estrogen implants in other brain sites. However, the maintenance of maternal behavior in the postpartum period and the gradual onset of maternal responsiveness in non-parturient females exposed to pups may both depend on mechanisms other than that critical to the immediate onset of maternal behavior at parturition. There- fore an investigation of the effects of MPO lesions on the immediate onset of maternal behavior associated with parturition is necessary for a complete understanding of the role of the MPO in the control of maternal behavior.

Normally when a rat gives birth, as each pup emerges she ingests the amnion, umbilicus, placenta, and accompanying fluids. This placentophagia is an integral part of maternal responsiveness during parturition.

If given the opportunity, some virgin rats spontaneously eat placenta obtained from donor females [4]. This nonparturitional placentophagia has been shown to be influenced by the estrous cycle in that virgins that ultimately eat placenta in all stages of the cycle will not have done so for the first time during proestrus [4]. In addition, it has been shown to be related to parturitional placentophagia in that experience with placenta during parturition alters subsequent response to placenta in nonpregnant females $[1,4]$.

The present study was designed to test the effects of medial preoptic (MPO) lesions on the responsiveness of parturient rats not only to pups and nesting material, and thus extend the findings of Numan, but also to placenta. In addition, the effects of MPO lesions on placentophagia in virgins was investigated.

To eliminate problems arising from performing stereotaxic surgery on pregnant animals, all animals were implanted with permanently indwelling bilateral electrodes aimed at the MPO, six to seven weeks prior to the production of the lesions. The preoptic region at which our elec-

${ }^{1}$ This research was supported by NSF Grant BNS76-04316 awarded to M.B.K.

${ }^{2}$ Reprint requests should be sent to Mark B. Kristal, Department of Psychology, SUNY-Buffalo, 4230 Ridge Lea Road, Buffalo NY 14226. 
trodes were aimed was the MPO of the Pellegrino and Cushman [9] atlas. This area corresponds to the nucleus preopticus periventricularis of the König and Klippel [3] atlas. Although the more lateral nucleus preopticus medialis is apparently the MPOA referred to in previous studies $[2,7,8$, 14] regardless of the atlas used for the published diagrams, we chose the more medial site because it contains a much higher density of estrogen-concentrating cells [10].

\section{METHOD}

\section{Animals}

Twenty-five female hooded rats (Charles River Breeding Laboratories), 4-6 months old and weighing 220-300 g, were used. Normal estrous cyclicity was verified by daily vaginal smears. Each rat was housed individually in a $24 \times 19 \times 18-\mathrm{cm}$ wire-mesh cage except during the last 2 wk of the experiment, when each was housed in a $45 \times 19 \times 25-\mathrm{cm}$ plastic cage with $3 \mathrm{~cm}$ of sawdust bedding. Food (Charles River Rat/ Mouse/Hamster Formula) and water were available ad lib. The rats were maintained on a $14 \mathrm{hr}$ on/10 hr off light cycle, with the on phase beginning at 6:00 am (EST).

\section{Placentophagia pretest}

After verification of normal cyclicity, a number of rats were given a pretest to determine whether they were placentophages or nonplacentophages. Each rat, after $2 \mathrm{hr}$ of food deprivation and $15 \mathrm{~min}$ of water deprivation, was presented with one donor placenta in a glass dish. Donor placentas were obtained surgically from $\mathrm{CO}_{2}$-killed primiparae on Day 21 of pregnancy. The placentas were then frozen, along with a few drops of normal saline, at $-20^{\circ} \mathrm{C}$, and stored until needed. At that time, the placentas were rapidly thawed and warmed to about $35^{\circ} \mathrm{C}$, and presented to the animals. The animal was given a 15 -min exposure to the dish of placenta. This procedure was repeated on 3 consecutive days, or until the rat ate the placenta. Placentophages are most likely to eat placenta on the first exposure; rats that have not eaten by the end of the third exposure are not likely to eat placenta at all [4]. Ten rats determined to be placentophages by this method were used as subjects in this study. Fifteen additional rats, purchased in the same batch and not pretested, served as placenta-naive animals.

\section{Procedure}

Surgery. Two to 3 weeks after the pretest, each rat underwent stereotaxic implantation of chronic MPO electrodes, under sodium pentobarbital anesthesia (Diabutal: 4 $\mathrm{mg} / 100 \mathrm{~g}$ body weight, IP) preceded by an injection of atropine sulfate $(0.08 \mathrm{mg} / 100 \mathrm{~g}$ body weight, IP). A pair of epoxylite-insulated, 0.012-in stainless steel electrodes (with the tips clipped off) was inserted according to the following coordinates: $1.7 \mathrm{~mm}$ anterior to bregma; $\pm 0.5 \mathrm{~mm}$ lateral to the middle of the superior sagittal sinus; $8.6 \mathrm{~mm}$ down from the skull [9]. Four stainless steel screws were used as anchors for the acrylic dental cement used to fasten the electrodes in place. The scalp was sutured around the dental cement and crystalline sulfathiazole was sprinkled on the incision. Each rat was allowed one week of recovery after the surgery, at which time estrous cyclicity was verified by daily vaginal smears.

Grouping. After surgery, depending on its pretest history, but otherwise randomly, each rat was assigned to one of 4 groups: Group NP-L (nonpregnant-lesion), 5 virgin placen- tophages to receive lesions $5 \mathrm{wk}$ after surgery and then presented on the next day with 5 donor placentas; Group NP-C (nonpregnant-control), 5 virgin placentophages to be subjected to a sham-lesion procedure $5 \mathrm{wk}$ after surgery and then presented on the next day with 5 donor placentas; Group P-L (pregnant-lesion), 10 placenta-naive females to be bred, given lesions on Day 21 of pregnancy (approximately 5 wk after surgery) and then observed and tested for subsequent maternal behavior; Group P-C (pregnant-control), 5 placenta-naive females to be bred but otherwise untreated through parturition, then observed and tested for subsequent maternal behavior. These Group P-C rats then served as controls for Group P-L. Five days after parturition the Group P-C rats received lesions and were then observed and tested for maternal behavior for 5 additional days. In this way, Group P-C served as an approximate replication of Numan's [7] procedure.

Breeding. Two weeks after surgery, each rat in Groups P-L and P-C were placed individually with a male. Daily vaginal smears were taken until the presence of sperm was detected, at which time the rat was considered to be in Day 1 of pregnancy. The next day (Day 2) she was returned to her home cage.

Lesion production. At noon on the day an animal was scheduled to receive lesions, she was removed from the colony room and placed under light ether anesthesia. A rectal cathode was inserted, then $1 \mathrm{~mA} \mathrm{dc}$ was passed for 6 seconds through each of the MPO electrodes. While still unconscious she was placed in a clean $45 \times 19 \times 25$-cm plastic cage with (a) 3 paper towels lining the floor of the cage, (b) a food hopper filled with $10 \times 2-\mathrm{cm}$ strips of paper, and (c) ad lib food and water. The sham-lesion procedure was identical except no current was passed. Paper towels, rather than more conventional bedding, were used to facilitate observations of location of placenta, feces, and urine in the cage.

Virgin placentophagia retest. At noon the day after undergoing the lesion or sham-lesion procedure, each rat in the nonpregnant groups (NP-L and NP-C) was presented, for 15 min, with 5 donor placentas in a glass dish, after which the number of placentas left was noted.

Maternal behavior-observation and testing. The day after receiving lesions, each rat in Groups P-L and P-C gave birth. Each rat was observed periodically throughout the day for the presence of a nest, the presence of placentas in the cage, and the responses made to the pups. The rat was otherwise undisturbed on the day of delivery.

From Day 1 postpartum until and including Day 5 postpartum, at noon each day, the quality of the nest, the location of the pups relative to the nest, and the location of all urine and feces relative to the nest were noted. Each rat was then moved, with her own litter, to a clean plastic cage lined with 3 paper towels and containing a food hopper filled with paper strips. The pups were then scattered about the cage. The mother was observed for the next $15 \mathrm{~min}$ and her behavior was noted, after which she was scored as: (a) had or had not yet finished retrieving her pups into a group, and (b) had or had not yet begun any nest-building activity.

After this test period on Day 5 postpartum, each rat in Group P-C received lesions and was then moved to a fresh cage with her litter. The rat was introduced into a fresh cage each day until and including Day 10 postpartum.

Histology. After testing was completed, each rat was anesthetized and perfused with normal saline followed by $10 \%$ Formalin. The brains were removed, fixed in Formalin, cut at $30 \mu$ on a freezing microtome, and stained with cresyl 
TABLE 1

MATERNAL RESPONSIVENESS ON THE DAY OF DELIVERY

\begin{tabular}{|c|c|c|c|c|c|}
\hline \multirow[t]{2}{*}{ Group } & \multicolumn{4}{|c|}{$\begin{array}{l}\text { Proportion of Group Showing Delay to: } \\
\text { (mean latency } \pm \text { SEM in hours) }\end{array}$} & \multirow{2}{*}{$\begin{array}{l}\text { Nest Absen } \\
\text { at Delivery }\end{array}$} \\
\hline & $\begin{array}{l}\text { Start Pup- } \\
\text { Licking }\end{array}$ & $\begin{array}{c}\text { Finish } \\
\text { Placentophagia }\end{array}$ & $\begin{array}{l}\text { Finish Pup- } \\
\text { Retrieval }\end{array}$ & $\begin{array}{c}\text { Start } \\
\text { Manipulating } \\
\text { Nest }\end{array}$ & \\
\hline $\begin{array}{l}\text { MPO Lesion } \\
\text { Before Delivery } \\
\quad(\text { Gp P-L, n=7) }\end{array}$ & $\begin{array}{c}0 / 7 \\
(0.00 \pm 0.00)\end{array}$ & $\begin{array}{c}3 / 7 \\
(2.86 \pm 1.46)\end{array}$ & $\begin{array}{c}5 / 7^{*} \\
(2.86 \pm 1.16)\end{array}$ & $\begin{array}{c}5 / 7^{*} \\
(3.57 \pm 1.31)\end{array}$ & $6 / 7+$ \\
\hline $\begin{array}{l}\text { No Lesion } \\
\text { Before Delivery } \\
\quad(\mathrm{Gp} P-\mathrm{C}, \mathrm{n}=5)\end{array}$ & $\begin{array}{c}0 / 5 \\
(0.00 \pm 0.00)\end{array}$ & $\begin{array}{c}1 / 5 \\
(0.20 \pm 0.22)\end{array}$ & $\begin{array}{c}0 / 5 \\
(0.00 \pm 0.00)\end{array}$ & $\begin{array}{c}0 / 5 \\
(0.00 \pm 0.00)\end{array}$ & $0 / 5$ \\
\hline
\end{tabular}

${ }^{*} p \leqslant 0.03$, Fisher exact probability test.
${ }_{\dagger} \leqslant \leqslant 0.005$. Fisher exact probability test.

violet. The extent and location of brain tissue damaged was then verified microscopically.

\section{RESULTS}

\section{Placentophagia in Virgins}

All of the animals in both Groups NP-L and NP-C immediately ate all 5 placentas presented to them in the retest. MPO lesions, therefore, had no effect on non-parturitional placentophagia as we measured it.

\section{Maternal Behavior on the Day of Parturition}

Two of the rats in Group P-L turned out upon histological examination to have had lesions that completely missed the hypothalamus. Their maternal behavior was normal. These 2 animals were therefore retroactively dropped from the study. Another Group P-L animal had her cranial implant loosened and pulled out prior to lesion production. Interestingly, at delivery she did show maternal behavior deficits: histological examination revealed that her MPO had been damaged probably by electrode movement as the electrodes pulled out. Nevertheless she was dropped from the study. This left 7 rats in Group P-L.

Table 1 contains maternal behavior latencies manifested on the day of delivery by the rats in Group P-L and Group P-C. Comparisions between experimental groups and their controls (proportions of groups), were made with the Fisher exact probability test, since the absence of variance in the raw data of the control groups precluded use of analysis of variance. The lesioned animals showed significantly fewer nests at birth, and significantly longer latencies to begin manipulating nest material and finish pup-retrieval. The lesioned and nonlesioned animals showed no statistically significant differences in the latencies to begin pup-licking or to finish placentophagia. It should be noted however, that although the groups did not differ statistically in regard to the latency to finish placentophagia, there were gross qualitative differences among the rats. Whereas only 1 nonlesioned rat did not eat placenta immediately (she took $1 \mathrm{hr}$ ), 3 of the 7 lesioned animals took in excess of $6 \mathrm{hr}$ to finish, and the rest showed no delay. The 3 were discrepant on other behavioral dimensions as well, enabling us to categorize the patterns of behavioral deficits in the lesioned group into two distinct syndromes.

Syndrome A. Three of seven lesioned animals showed delayed placentophagia at parturition. These animals demonstrated long latencies to all maternal behaviors, except licking, at the time of delivery. Typically, they walked about as they gave birth leaving the pups and placentas where they fell. As they walked about, when they encountered a pup, a placenta, or a section of paper towel soaked with birth fluid they stopped to lick. Sometimes they chewed on the umbilicus, separating the pup from the placenta, but typically made no efforts at pup-retrieval or placentophagia. They did no nest-building before or during delivery. By an average of $6.6 \mathrm{hr}$ after the last pup emerged, however, they had consumed all the placenta (except for one rat that never consumed all the placenta and that was assigned as arbitrary latency score equal to that of the next longest latency, $7 \mathrm{hr}$ ), and by $24 \mathrm{hr}$ they had constructed a nest around their litters. This is in contrast to all 5 animals in Group P-C, which built nests before delivery, gave birth in the nest or retrieved immediately, and typically consumed all of their placentas immediately as they emerged.

Syndrome B. In most respects, the 4 remaining Group P-L animals showed normal maternal behavior (immediate licking, immediate or rapid retrieval, immediate placentophagia) but exhibited impaired nest-building. Two of them appeared to make immediate efforts at nest construction, i.e., they manipulated (threaded, carried, pushed) the paper towels and strips, but their efforts appeared disorganized and undirected, and were largely ineffective. Only 1 of these produced even a rough pile of papers and was thus scored as having a nest present at birth. The other 2 rats made no efforts at all at nest-building until some hours after the last pup emerged. Twenty-four hr after delivery all 4 of these rats were observed to have constructed nests around their pups, but these nests were of poorer quality (smaller, looser, less complete, etc.) than the nests constructed by the Group P-C animals.

\section{Maternal Behavior on Postpartum Days 1-5}

For each animal the percentage of postpartum days 1-5 
TABLE 2

MATERNAL RESPONSIVENESS OF GROUP P-C RATS $(\mathrm{N}=5)$ BEFORE AND AFTER MPO LESIONS

\begin{tabular}{lcc}
\hline & $\begin{array}{c}\text { Mean Percent }( \pm \text { SEM) of Days: } \\
\text { on which retrieval } \\
\text { was finished within } \\
15 \mathrm{~min}\end{array}$ & $\begin{array}{c}\text { on which nest } \\
\text { material } \\
\text { manipulation was } \\
\text { started within } \\
15 \text { min }\end{array}$ \\
\hline $\begin{array}{l}\text { Postpartum Days 1-5 } \\
\text { (Before Lesion) }\end{array}$ & $92.0 \pm 8.00$ & $84.0 \pm 11.66$ \\
$\begin{array}{l}\text { Postpartum Days 6-10 } \\
\text { (After Lesion) }\end{array}$ & $44.0 \pm 14.70$ & $20.0 \pm 8.94$ \\
Results of 2-way ANOVA \\
(Days $\times$ Animal with $\mathrm{df}=1,4)$
\end{tabular}

on which nest-material manipulation was started within the 15-min test period was computed. These data were analyzed using a 1-way ANOVA, which revealed that the animals with lesions did not differ significantly from controls (Gr. P-L: $\bar{X}=60.71$ : Gr. P-C: $\bar{X}=84.00: F(1,10)=2.75, p>0.05$ ). Throughout Days $1-5$, the Syndrome B rats continued to build relatively poor quality nests (i.e., smaller, looser) just as they had on the day of delivery. Syndrome A rats on the other hand, although considerably delayed, (within 15-min on only $43 \%$ of the test days) tended to build nests of normal quality each day.

A similar comparison of the percentage of days on which pup-retrieval was finished within the 15 -min test period revealed no statistically significant impairment for the lesioned group. (Gr. P-L: $\bar{X}=85.00$ : Gr. P-C: $\bar{X}=92.00$ : $F(1,10<<1)$. The rats of both sub-groups appeared to manifest fairly normal pup-oriented behaviors (retrieving, nursing, licking).

One other behavior that is likely a characteristic of maternal responsiveness in the rat, could very clearly be observed. The paper towels in the cage made it very easy to see where the rats had urinated and defecated during the preceding $24 \mathrm{hr}$. The nonpregnant animals in Groups NP-L and NP-C clearly voided throughout their cages, with a possible tendency to avoid their small $(10-\mathrm{cm}$ square) sleeping mats. Maternal rats very clearly did not defecate or urinate in the entire half of the cage that contained the pups. In other words, after $24 \mathrm{hr}$ in a cage, the pups would be in a nest on one side of the cage and all of the urine and feces would be near the far wall at the other side of the cage. All of the animals in Groups P-L and P-C behaved in this manner both before and after receiving lesions.

Maternal Behavior After Lesion on Day 5

The 5 rats in Group P-C received lesions on Day 5 postpartum. Table 2 summarizes and contrasts their maternal behavior before receiving the lesions with their maternal behavior after receiving the lesions. Latencies to both retrieving and nest-building were significantly longer after receiving the lesion.

A note of caution is necessary regarding the data on retrieval, however. Since each rat was continuously tested with her own litter, the pups were 6-10 days old during the post-lesion observation. After being scattered about the new cage these pups would often crawl unaided into a neat pile in a matter of minutes. Since the mother usually did nothing to help, this was scored as a failure to retrieve. It is possible however that these mothers would have gotten around to retrieving the pups within the 15 min test period if the pups had remained scattered.

It should be emphasized that no animal in any condition failed to act maternally. All of the rats built nests, licked and nursed their pups, etc. Almost all of the pups born to all groups survived (the first 2 pups delivered by one of the rats in Group P-C and the first pup delivered by a rat in Group P-L died, possibly as a result of damage caused by the rectal cathode) and all that did survive appeared quite healthy. The differences between the groups were primarily a matter of latency.

\section{Histology}

Diagrammatic representations of the minimum and maximum amounts of damage of both Syndrome A and Syndrome $\mathrm{B}$ animals are presented in Fig. 1.

The lesions tended to be smaller and more medial than those depicted in the Numan study ([7] Fig. 1). According to the atlas of Pellegrino and Cushman [9], the lesions would be described as located in the area referred to as the medial preoptic area (MPO) and as extending into the medial aspect of the structure referred to as the preoptic area (POA). According to the atlas of König and Klippel [3], however, the lesions would be described as having produced the greatest amount of damage to the nucleus preopticus periventricularis (pop), with most extending laterally to include the medial portion of the nucleus preopticus medialis (pom).

The lesions in those animals that exhibited Syndrome A tended to be (a) quite variable, and (b) more anterior in extent, often extending to the level of the diagonal band of Broca. The lesions in those animals exhibiting Syndrome B tended to be (a) less variable, and (b) more posterior in extent, often including portions of the arcuate nucleus.

\section{DISCUSSION}

Nest-building (particularly its initiation) was the component of maternal behavior most consistently affected by the MPO lesions in the present experiment. The initiation of pup retrieval, nursing and placentophagia were also disrupted at 
A

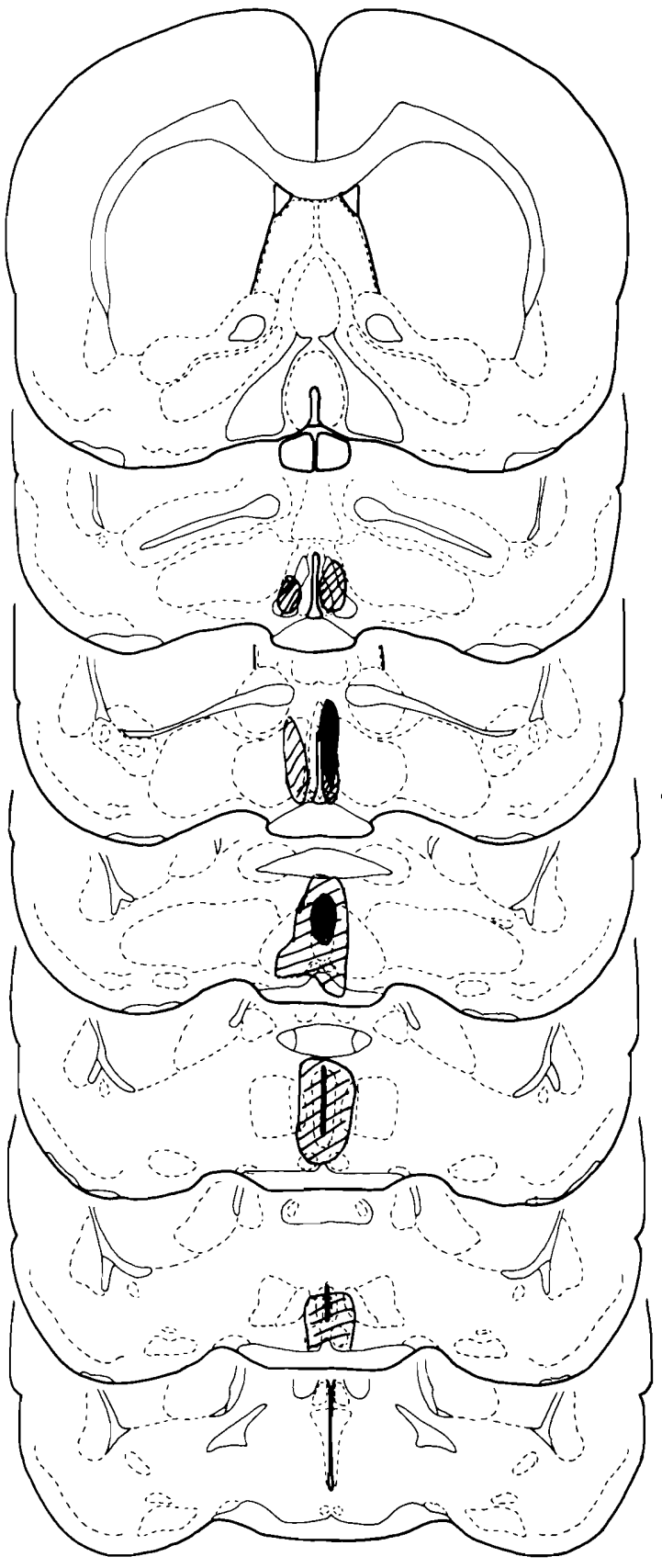

B

A P

8.2

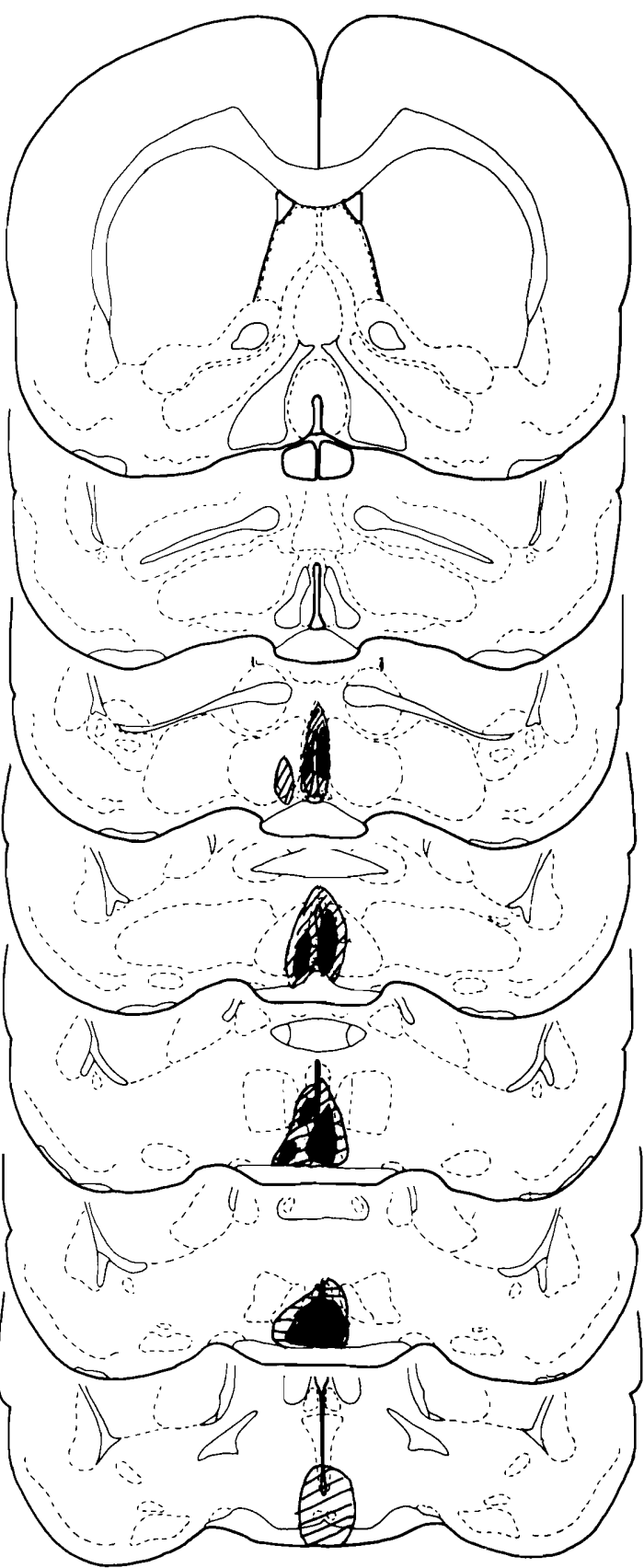

FIG. 1. Diagrammatic representation of lesions in rats manifesting either Syndrome A or B, showing the area of destruction common to all animals (black) and the areas destroyed in some, but not all animals (hatched) (from Pellegrino and Cushman, 1967). 
parturition, but only in three of seven animals with lesions.

Although placenta-eating was delayed in nearly half of the parturient rats with lesions, pup-licking, presumably accompanied by amniotic fluid ingestion, appeared immediately at delivery in all animals. Numan ([7] p. 750) mentioned that his MPOA animals, although deficient in most maternal behavjors, also licked their pups. This distinction between the ingestion of birth fluid and of placenta is of interest since they appear in the normal parturient rat as part of a single ingestive sequence.

Placentophagia during parturition has been shown to influence subsequent responses to donor placenta by experienced non-parturient rats $[1,4]$. In contrast to this indicated association between the two, MPO lesions, as produced in this experiment, disrupted placentophagia during delivery but had no effect on placentophagia in our virgin animals. The difference in effect of MPO lesions on placentophagia during parturition and on response to placenta by nonpregnant rats, coupled with some evidence presented in previous studies $[1,6]$, raises the question of the extent to which parturitional placentophagia and nonpregnant placentophagia are distinct phenomena.

The effects of the lesions we performed on Day 5 postpartum, i.e., impaired nest-building and pup-retrieval, agree with the results reported by Numan [7] except in degree of impairment. Whereas our animals showed delays in nest-building and retrieval, Numan reported almost complete elimination of these two behaviors, and of nursing as well. We noticed no effect of MPO lesions on nursing after the day of production of the lesion. However, since we did not rigorously focus on and measure nursing. it is possible we overlooked some slight impairment. Since the anteriorposterior extent of the lesions in the present study are comparable to those reported in the Numan [7] study, the difference in severity of deficit in our animals and in Numan's animals may be attributable to (a) the more medial placement of our electrodes, (b) differences between the effects of lesions produced through indwelling electrodes and those produced through acutely inserted electrodes, or (c) strain differences. We felt that it is unlikely that testing daily in new cages, in contrast to Numan's procedure of testing repeatedly in the same cage, contributed to the difference in results.

Our animals that received lesions before delivery showed less impairment of nest-building and retrieving on Days 1-5 than our Day-5 lesion animals showed on Days 6-10. This is somewhat surprising since it is typically easier to disrupt the initiation of maternal behavior than it is to disrupt its maintenance once established [11]. It may be that the MPO plays a greater role in the maintenance than in the initiation of maternal behavior.

The results of our experiment, in general, suggest independent mechanisms for the various behavioral components of maternal behavior. For example, whereas our lesions produced measureable impairments in many maternal behavior measures, no effect at all was found on pup-licking or on the location of waste excretion (away from the gathered pups). Presumably the neural substrate of these behaviors lies entirely outside the damaged areas. Within the general area of damage, some lesions interfered with placentophagia, for example, while others did not. Some lesions impaired nest building only, leaving the other maternal behavior components unaffected. Although the behavioral impairments seemed to fall into two easily discriminable patterns, one characterized by deficits in placentophagia, retrieval and nest-building, and the other characterized by a deficit only in nest-building, differences between the groups in the characteristics of the neural damage could not be distinguished conclusively on the basis of our histological examination.

\section{REFERENCES}

1. Engwall, D. B. and M. B. Kristal. Placentophagia in rats is modifiable by taste aversion conditioning. Physiol. Behat. 18: 495-502, 1977.

2. Ho, G. K., D. Quadagno, and H. Moltz. Intracranial cycloheximide: Effects on maternal behavior in the postpartum rat. Pharmac. Bioch'sm. Behar. 2: 455-458, 1974.

3. König, J. F. R. and R. A. Klippel. The Rat Brain: A Stereotaxic Atlas. Huntington, N. Y.: Robert E. Krieger, 1963.

4. Kristal, M. B. and G. C. Graber. Placentophagia in nonpregnant rats: Influence of estrous cycle stage and birthplace. Physiol. Behav. 17: 599-605, 1976.

5. Moltz, H., M. Lubin, M. Leon and M. Numan. Hormonal induction of maternal behavior in the ovariectomized nulliparous rat. Phusiol. Behav. 5: 1373-1377, 1970.

6. Noonan, M. and M. B. Kristal. Nonparturitional exposure to donor placenta and placentophagia after lateral hypothalamic lesions in rats. Bull. Psychon. Soc. 12: 140-142, 1978.

7. Numan, M. Medial preoptic area and maternal behavior in the female rat. J. comp physiol. Psychol. 87: 746-759, 1974.

8. Numan, M., J. S. Rosenblatt and B. R. Komisaruk. Medial preoptic area and onset of maternal behavior in the rat. J. comp. physiol. Psychol. 91: 146-164, 1977.

9. Pellegrino, L. J. and A. J. Cushman. A Stereotaxic Atlas of the Rat Brain. New York: Appleton-Century Crofts, 1967.
10. Pfaff, D. and M. Keiner. Atlas of estradiol concentrating cells in the central nervous system of the female rat. J. comp. Neurol. 151: $121-158,1973$.

11. Rosenblatt, J. S. Views on the onset and maintenance of maternal behavior in the rat. In: Development and Evolution of Behavior, edited by L. R. Aronson, E. Tobach, D. S. Lehrman, and J. S. Rosenblatt. San Francisco: W. H. Freeman, 1970.

12. Siegel, H. I. and J. S. Rosenblatt. Estrogen-induced maternal behavior in hysterectomized-ovariectomized virgin rats. Plysiol. Behav. 14: 465-471, 1975.

13. Siegel, H. I. and J. S. Rosenblatt. Latency and duration of estrogen induction of maternal behavior in hysterectomizedovariectomized virgin rats: Effects of pup stimulation. Physiol. Behav. 14: 473-476, 1975.

14. Smotherman, W. P., J. W. Hennessy and S. Levine. Medial preoptic area knife cuts in the lactating female rat: Effects on maternal behavior and pituitary-adrenal activity. Physiol. Psychol. 5: 243-246, 1977.

15. Terkel, J., and J. S. Rosenblatt. Humoral factors underlying maternal behavior at parturition: Cross transfusion between freely moving rats. J. comp. physiol. Psychol. 80: 365-371, 1972. 